\title{
Differential diagnosis of acute traumatic hip pain in the elderly
}

\author{
Matthew Hampton, Richard Stevens, Adrian Highland, Richard Gibson, Mark B. Davies
}

From the Sheffield Teaching Hospitals NHS Foundation trust, Northern General Hospital, Sheffield, Herries Road, AU

Elderly patients who present with an inability to weight bear following a fall, with normal radiographs, should be appropriately investigated to rule out an occult hip fracture (OHF). We aim to identify both the range and incidence of the differential diagnosis of acute traumatic hip pain in a large series of patients investigated for $\mathrm{OHF}$.

A retrospective analysis of consecutive patients investigated for an OHF with magnetic resonance imaging (MRI) was performed. Dedicated musculoskeletal radiologists reported the MRI scans. All diagnoses including hip fractures, other fractures and soft tissue injuries were recorded. Case notes were reviewed for all patients to identify subsequent complications, management and outcomes.

A total of $\mathbf{1 5 7}$ patients fulfilled the inclusion criteria. $52(33 \%)$ patients had a fracture of the proximal femur. The majority of patients with proximal femoral fractures required surgical intervention. 9 patients who had fractures of the greater trochanter of the femur without fracture extension across the femoral neck were managed non-operatively.

$40(25 \%)$ patients had fractures of the pelvis, with a combined pubic rami and sacral fracture occurring frequently. The most common diagnosis was a soft tissue injury alone that was seen in $60(38 \%)$ patients imaged. Injuries to the gluteal muscle group, iliopsaos complex and trochanteric bursa were most prevalent. All patients with soft tissue injuries or fractures of the pelvis were successfully managed non-operatively.

This study highlights a wide range of differential diagnoses in elderly patients presenting with acute traumatic hip pain. The proximal femur was fractured in $33 \%$ of patients imaged for OHFs in our series. The most common diagnosis was a soft tissue

No benefits or funds were received in support of this study. None of the authors have a conflict of interest. injury around the hip and pelvis; these injuries can be successfully managed without surgery.

Keywords: Hip pain; hip fracture; pelvic trauma; MRI ; occult hip fracture.

\section{INTRODUCTION}

An occult hip fracture (OHF) is the single most likely diagnosis in any elderly patient who presents with a history and examination that is suggestive of a hip fracture but the plain radiographic assessment appears normal $(1,2)$. The typical features on clinical assessment often reveals a patient with groin pain on active hip movements and pain or an inability to bear weight. Passive hip movements may recreate the groin pain ; there may be pain on straight leg raising or an inability to do so ; or pain on passive rotation and axially loading of the hip (3).

\footnotetext{
Matthew Hampton,

Richard Stevens,

Adrian Highland,

Richard Gibson,

Mark B. Davies

Sheffield Teaching Hospitals NHS Foundation trust, Northern General Hospital, Sheffield, Herries Road, AU

Correspondence : Matthew Hampton, 07786882453, Sheffield

Teaching Hospitals NHS Foundation trust, Northern General Hospital, Herries Road, Sheffield S5 7AU. Phone : 01142434343

Email : Matthew.hampton@nhs.net

○ 2021, Acta Orthopædica Belgica.
} 
Anterior-posterior (AP) and lateral plain radiographs are obligatory but if there is no evidence of a fracture, then further imaging with magnetic resonance imaging (MRI) should be obtained. In the United Kingdom, this is mandated within 24 hours as per the National Institute for Health and Care Excellence (NIHCE) guidelines (4).

The prevalence of OHF is reported to be between $2-10 \%(5,6)$. Previously, MRI has been reported to be $100 \%$ accurate in the diagnosis of an occult hip fracture (7). While CT may be quicker \& easier to obtain, and in some studies demonstrated equal efficacy at identifying fractures (8) MRI offers the additional benefit of diagnosing alternative soft tissue pathology (9). The MRI findings in this patient population are important to recognise as it is important to correctly treat any fractures and to offer safe rehabilitation of patients with other injuries. While imaging of fragility fractures and soft tissue injuries around the hip is established $(8,9,10)$ there is a paucity of evidence within the literature regarding the incidence of differential diagnoses when MR imaging is requested to exclude OHF following low energy falls in the elderly. Therefore, the aim of this study was to establish the range and incidence of differential diagnoses of acute traumatic hip pain in a large series of patients investigated for occult hip fractures. Many of the diagnoses we encounter in this study would not be identified using computer tomography (CT) scanning alone. Our findings could help guide clinicians to the appropriate diagnosis in elderly patients with acute hip pain when CT scans are used instead of MRI to investigate for OHFs

\section{MATERIALS AND METHODS}

All patients presented to a single major trauma centre within the United Kingdom (UK) between January 2014 and June 2017. The inclusion criteria were an elderly patient, presenting to our unit with acute hip pain secondary to a history of trauma and in whom plain radiographic imaging (including orthogonal views) of the pelvis and hip had failed to establish a diagnosis.

Exclusion criteria were those patients with a known malignancy and suspected pelvic or proximal femoral metastases, radiographically diagnosed stress fractures with no history of a preceding traumatic injury and those with a clear cause of the hip pain from the plain radiographs.

A retrospective notes review allowed collation of patient demographics as well as a review of the full clinical presentation. Review of the picture archiving a system (PACS) permitted a review of the MRI reports, all of which were performed by dedicated musculo-skeletal radiologists. Subsequent management was also reviewed together with any recorded complications.

\section{RESULTS}

Between January 2014 and June 2017, a total of 226 patients, between 68-94 years of age, were identified who underwent an MRI scan for suspected OHF. Of these, 69 patients were excluded from this study. 36 patients were excluded due to requiring further investigations for suspected metastatic disease, 18 patients presented with suspected stress fractures of their proximal femur and 15 patients had severe osteoarthritis (OA) on their MRI scans with associated joint effusions which was the only finding which could account for their symptoms. The OA was evident on plain radiographs prior to the MRI so they were also excluded.

157 patients presented who fulfilled the inclusion criteria and their MRI diagnoses were included in this study (Graph. 1).

Of the 157 patients imaged, 52 (33\%) were diagnosed with fracture of proximal femur. Within this group of patients, 19 had intracapsular fractures, there were 15 with intertrochanteric fractures and 18 had fractures of the greater trochanter. Of

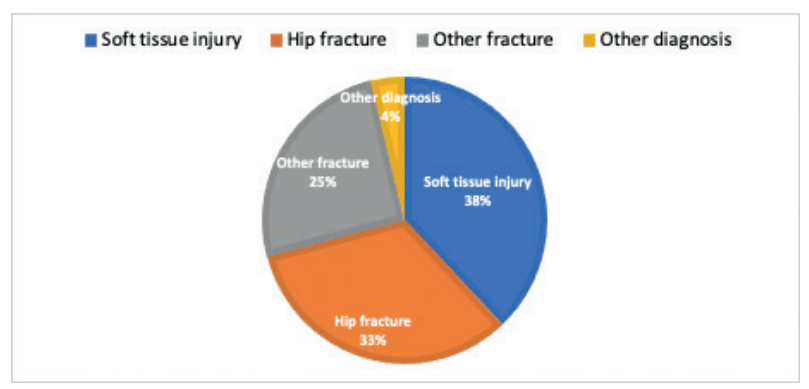

Graph 1. - Pie chart showing the frequency of each diagnosis. It shows that a soft tissue injury alone is the most common diagnosis. 
Table 1. - Table listing the different locations of hip fractures identified on MRI scan and associated frequency

\begin{tabular}{|l|c|}
\hline Hip fracture location & Total number \\
\hline Intracapsular & 19 \\
\hline Extracapsular & 15 \\
\hline $\begin{array}{l}\text { Greater trochanter - intertrochanteric } \\
\text { extension }\end{array}$ & 9 \\
\hline $\begin{array}{l}\text { Greater trochanter - No intertrochanteric } \\
\text { extension }\end{array}$ & 9 \\
\hline
\end{tabular}

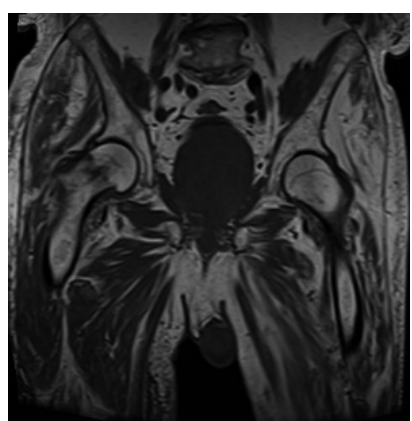

Figure 1a. - Large FOV coronal T1 TSE, showing a serpiginous intracapsular fracture of the right femoral neck. The large field of view allows screening for other pelvic fractures and para-ischial soft tissue injuries

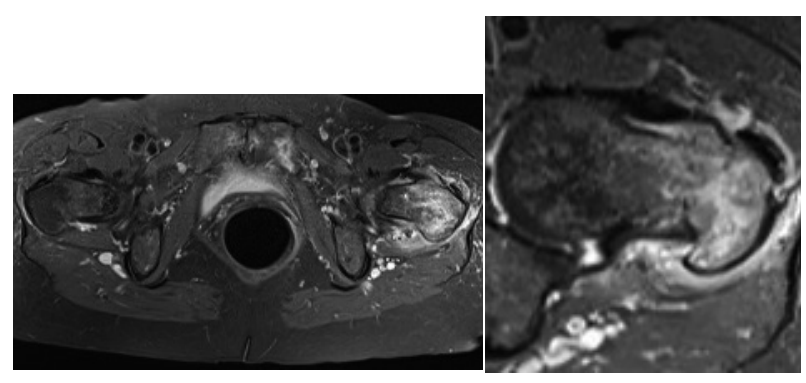

Figure 1b. - Axial PD TSE with fat suppression showing diffuse oedema surrounding an extracapsular fracture of the left femoral neck. There is an additional fracture of the medial aspect of left superior pubic ramus.

those patients with greater trochanter fractures, in 9 patients the fracture line extended across the femoral neck and 9 patients had avulsion fractures with no propagation of the fracture lines (Table 1).

All 19 patients who were diagnosed with an intracapsular femoral neck fracture proceeded to surgical intervention (Fig 2) with 12 patients undergoing cannulated hip screw fixation and 2 patients dynamic hip screw (DHS) fixation. In 4 patients, the intracapsular hip fracture had displaced in the interval between the initial plain

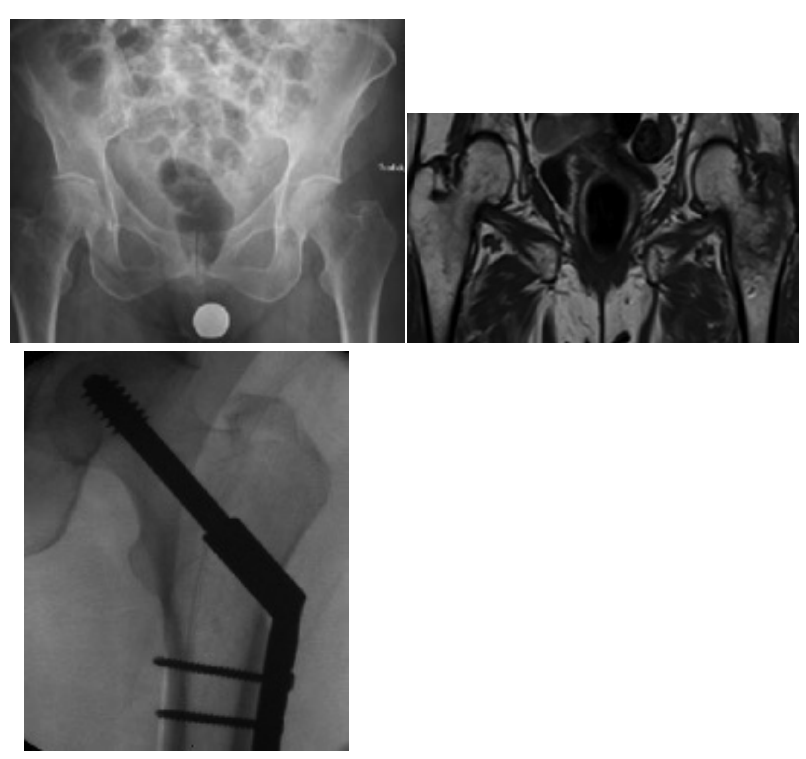

Figure 2. - Plain radiographs and subsequent coronal T1 SE MRI, which reveals medial extension of a greater trochanter fracture across the inter-trochanteric region. The patient was treated with surgical fixation using a DHS.
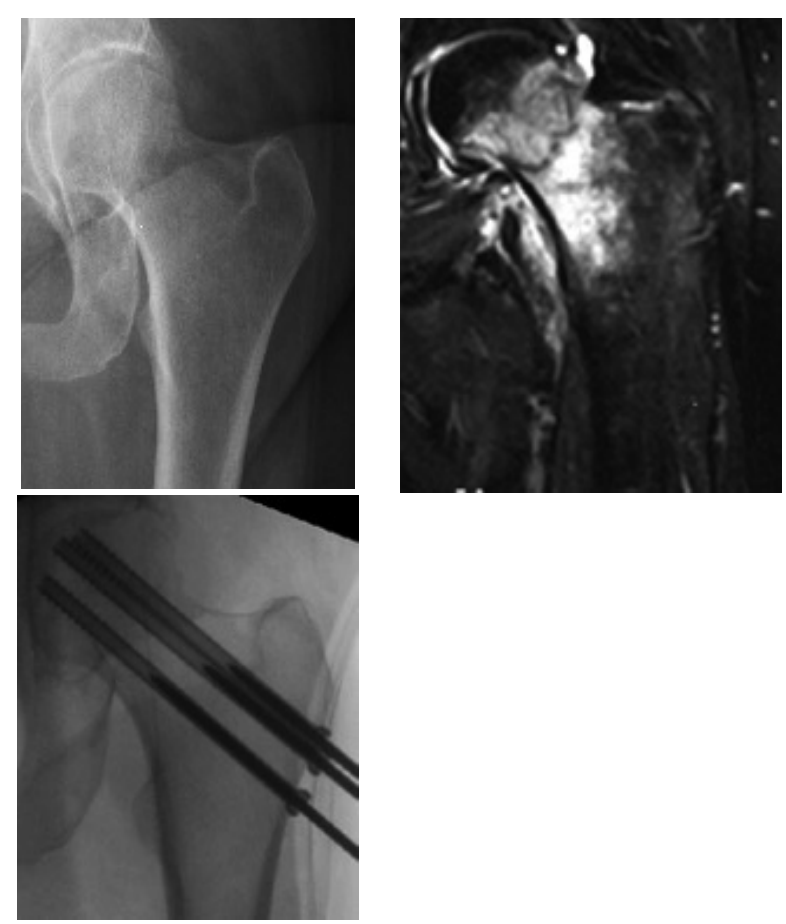

Figure 3. - Patient presented following a fall from standing with an inability to weight bear, pain on straight leg raise, axial loading and passive rotation of the hip. Plain radiographs didn't reveal a fracture however the subsequent Coronal T2 with fat saturation MRI revealed an un-displaced sub-capital fracture. The patient was treated with cannulated hip screws. 
radiographic assessment on admission and they underwent cemented hip hemiarthroplasty. The time interval between normal radiographs and MRI scan was prolonged in all 4 patients, ranging from 3249 hours. A single patient was treated with a long cephalomedullary femoral nail as the MRI revealed previously unsuspected femoral metastases from primary breast carcinoma.

A fracture of the greater trochanter was revealed on MRI in 18 patients. The imaging distinguished whether or not the fracture extended across the femoral neck or intertrochanteric region in 9 of these patients $(50 \%)$. Given that these patients were thought to be at significant risk of fracture propagation and displacement if managed nonoperatively, all of the patients with extension were treated with fixation with DHS (Fig 3). In the remaining group of patients with avulsion fractures of the greater trochanter, the MRI findings allowed subsequent non-operative management with protected toe touch weight bearing if able for a total of 6 weeks. None of these fractures had further fracture displacement requiring intervention.

All 15 patients diagnosed with intertrochanteric fractures of the femoral neck were treated with DHS fixation.

$40(25 \%)$ patients sustained other fractures that accounted for their presentation (Table 2). These included 30 patients diagnosed with fractures of the pubic ramus. In 25 of these patients, concomitant fractures of the sacrum and sacral ala were established. These fractures were lateral compression type 1 fractures as per the young and burgess classification. All of these patients were managed non-operatively with analgesia, regular physiotherapy and permitted to bear weight fully. In each case, referral for metabolic bone services for investigation and treatment of osteoporosis was made.

Similarly, a fracture of the acetabulum was revealed in 8 patients, typically involving the anterior acetabulum caused by femoral head impaction during the fall. These fractures were not displaced or evident on pain radiographs. All these patients were also managed non-operatively and permitted to full weight bear as able.

In $60(38 \%)$ patients, isolated soft tissue injuries accounted for the most frequent group of diagnoses
Table 2. - Other fractures identified on MRI scan; pubic rami fractures frequently have posterior sacral fractures associated with them

\begin{tabular}{|l|c|}
\hline Other fracture location & Total number \\
\hline Pubic ramus + sacrum & 25 \\
\hline Pubic ramus alone & 5 \\
\hline Acetabulum & 8 \\
\hline Pubic symphysis & 1 \\
\hline
\end{tabular}

Table 3. - Soft tissue injury was the most common diagnosis on MRI scans. These injuries can be successfully managed conservatively

\begin{tabular}{|l|c|}
\hline Soft tissue injury & Total number \\
\hline Gluteal muscles & 16 \\
\hline Iliopsoas complex & 14 \\
\hline Trochanteric bursa & 12 \\
\hline Adductor muscles & 7 \\
\hline Subcutaneous haematoma & 4 \\
\hline External rotators & 2 \\
\hline Hamstrings & 2 \\
\hline Proximal quadriceps & 1 \\
\hline
\end{tabular}

in this study (Table 3). The three anatomic areas around the hip that were most commonly injured included the gluteal muscles, the iliopsoas complex and the trochanteric bursa, constituting $70 \%$ of the soft tissue injuries. 16 patients sustained a tear to the gluteal muscles evidenced by discontinuity of the musculotendinous unit or significant oedema on the MRI. In the 14 patients with injuries to the iliopsoas complex, 5 patients had avulsed the tendon from the lesser trochanter of the femur without any evidence of fracture and 9 patients had a torn or inflamed iliopsoas tendon. 12 patients either had fluid or haematoma within the trochanteric bursa.

Other soft tissue injuries identified from the MRI scan are listed in table 3. One patient had a psoas collection, most likely from an infective process, this was treated with antibiotics and with further clinical and MRI follow up, it subsequently resolved without the need for surgical intervention. One patient had evidence of extensive muscle oedema in the gluteal muscles and thigh compartments on the MRI scan. This was secondary to a prolonged period of lying on the floor following a fall. This patient developed rhabdomyolysis and required haemodialysis. 


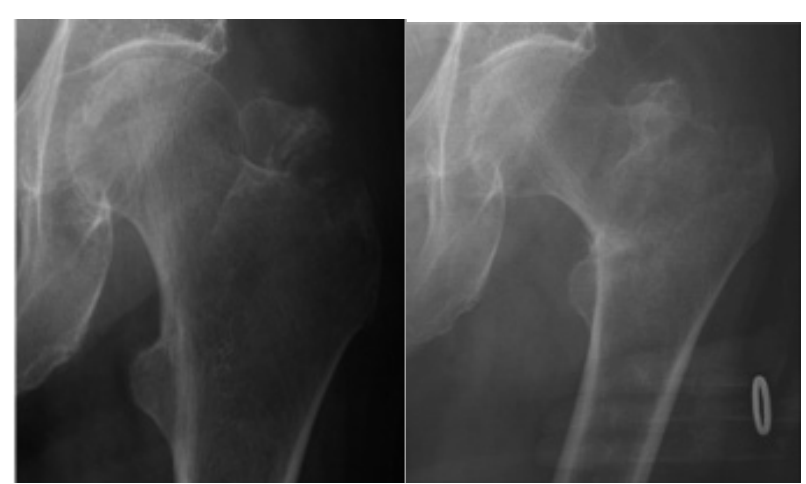

Figure 4. - Radiagropraphs showing subsequent displacement of a greater trochanter fracture treated non-operatively.

All patients with soft tissue injury were managed conservatively with analgesics and early weight bearing. None required surgical intervention.

One patient had a history of fall and the MRI scan showed early avascular necrosis of the femoral head, which was idiopathic in aetiology, this patient was acutely managed with analgesia and physiotherapy and went on to receive an outpatient total hip replacement. In 5 patients, no clear diagnosis could be made neither from the plain radiographs nor from the MRI scans of the hip and pelvis. Within this group of patients, 3 had subsequent MRI of the lumbo-sacral spine that identified a positive spinal pathology and this was attributed to the cause of their hip symptoms. Two patients were diagnosed with spinal stenosis and one with a prolapsed intervertebral disc at $\mathrm{L} 4 / 5$ intervertebral level causing L5 nerve root irritation - this patient had a nerve root injection to settle their symptoms and allow physiotherapy. Finally, in the remaining two patients with both normal plain radiography and MRI scans, no formal diagnosis for their symptoms was made. No further investigations were issued and they both were mobilised by physiotherapy with analgesia and discharge home when the pain settled.

\section{DISCUSSION}

MRI is superior to plain radiographs, computer typography (CT) and bone scintigraphy for the early detection of an occult hip fracture $(7,14)$. Additionally, as well as being $100 \%$ specific and sensitive for the detection of a hip fracture, it is also a sensitive test for identifying other pathologies. In the UK, NIHCE guidance recommends that MRI should be obtained within 24 hours from the time of raising a clinical suspicion of OHF. This present study shows that in patients who present with a clinical picture suggestive of a hip fracture but normal radiographs, 33\% will have a fracture of the proximal femur, supporting the evidence that led to the NIHCE guidelines. With $27 \%$ of patients imaged requiring hip fracture surgery, it is important to nurse these patients with care, restricting weight bearing until an MRI excludes a diagnosis of hip fracture (15). In the 4 cases of fracture displacement from time of radiographs to surgery seen in this study, this resulted in a change in the surgical management from fixation, and salvage of their native femoral head, to prosthetic replacement surgery.

The study also confirms the benefit of MRI in identifying fracture configurations especially with fractures of the greater trochanter (7). With fracture line propagation extending across the intertrochanteric region, surgical fixation can be undertaken confirming the findings from other studies (13). In isolated avulsion fractures of the greater trochanter, we demonstrated that MRI allowed definitive planning of non-operative treatment.

$83 \%$ of patients with pubic rami fractures had posterior pelvic fractures in our study, consistent with work by Scheyerer et al and Cosker et al $(18,10)$. These were identified as insufficiency fractures. The patients with complete pelvic ring injuries in this series had prolonged inpatient admissions and often failed to return to their own accommodation on discharge (19).

Patients with suspected insufficiency fractures should have an early referral to a metabolic bone unit for investigation of osteoporosis as the majority of these fractures are secondary to low bone density and secondary prevention may be of significant benefit (20). Pain can last 6-8 weeks and may persist longer. Stabilisation significantly improves pain intensity and will allow improved mobilisation when the sacrum is fractured. Percutaneous stabilisation may be considered in certain patients who have persistent pain and instability although this was 
not considered necessary in the present study due to the ability of the patients to bear weight prior to discharge from hospital $(21,22)$.

The most common diagnosis in our patients was a soft tissue injury with no fracture (38\%), with injury to the gluteal muscles, iliopsoas tendon and trochanteric bursa being most prevalent. The true prevalence of soft tissue injuries in low energy falls in the elderly is likely to be far higher. No gold standard exists for investigation of these injuries, and those with symptoms severe enough to warrant an MRI likely represent a subgroup of the more severe injuries. This under-diagnosis reflects that the majority of these injuries can be managed non-operatively regardless of the exact cause, and therefore an MRI may not be warranted. However, early, accurate diagnosis can permit confident, early rehabilitation with understandable benefits to this age group of patients. Intuitively, there may be a reduction in the length of hospital stay, but this cannot be proven for the present study. Previous studies have illustrated delays in the diagnosis of soft tissue injuries following trauma in which CT was used for the initial investigation for suspected OHF (23). The accuracy of an MRI scan to detect tears of the abductor tendons is $91 \%$ (24). When identified the vast majority can be managed nonoperatively, as seen in this series, however a small number of patients with persistent pain and weakness may require surgical reconstruction using a variety of techniques described in the literature $(25,26)$. The evidence base and current literature for the identification and management of tears or avulsion injuries to the iliopsoas tendon complex is limited. It is an important differential of traumatic hip pain in the elderly as non-operative management is effective with a favourable prognosis $(27,28)$. In our series we identified 14 patients who sustained injuries to the iliopsoas complex alone, all of which were successfully managed without the need for surgical intervention.

This study does have limitations. The retrospective nature of the study meant that management and outcomes of patients could only be identified up until the point of discharge from the trauma centre. Patients included in this evaluation of the differential diagnosis of traumatic hip pain were subject to selection bias as only patients who underwent MRI scans were included. There are, of course, patients who presented to the unit with traumatic hip pain who didn't require an MRI scan to form a diagnosis and these patients were not studied.

\section{CONCLUSION}

MRI is a highly sensitive investigation and will identify a wide range of differential diagnoses. The most likely diagnosis is a simple soft tissue injury, which can be managed conservatively. It may also be used to separate proximal femoral fractures that may be treated conservatively, (e.g. avulsion of the greater trochanter without extension), from fractures that may be treated with internal fixation.

The present study identifies a previously unquantified but wide range of pathologies that account for the acutely painful hip in an elderly population who sustained trauma. The study is useful in highlighting the need for MRI in governing further management of patients presenting in this manner.

\section{REFERENCES}

1. Braithwaite RS, Col NF, Wong JB. Estimating hip fracture morbidity, mortality and costs. J. Am. Geriatr. Soc. 2003 Mar ; 51(3) : 364-70.

2. Egol KA, Koval KJ, Kummer F, Frankel VH. Stress fractures of the femoral neck. Clinical orthopaedics and related research $1998 \mathrm{Mar}$; (348) : 72-8.

3. LeBlanc KE, Muncie HL Jr, LeBlanc LL. Hip fracture : diagnosis, treatment, and secondary prevention. AM Fam. Physician 2014 Jun 15 ; 89(12) : 945-51.

4. Management of hip fracture in adults : summary of NICE guidance. BMJ 2011; 342 doi : https://doi.org/10.1136/ bmj.d3304 (Published 21 June 2011).

5. Dominguez S, Liu P, Roberts C, Mandell M, Richman PB. Prevalence of traumatic hip and pelvic fractures in patients with suspected hip fracture and negative initial standard radiographs-a studyof emergency department patients. Acad. Emerg. Med. 2005 ; 12 : 366-9.

6. Y. Beloosesky, A. Hershkovitz, A. Guz, H. Golan, M. Salai, A. Weiss. Clinical characteristics and long-term mortality of occult hip fracture elderly patients. Injury, $2010 ; 41(4): 343-347$.

7. Karen M. Verbeeten, Kirstine Lintrup Hermann, Maria Hasselqvist, Gunnar S. Lausten, Per Joergensen, Claus Munk Jensen, Henrik S. Thomsen. The advantages of MRI in the detection of occult hip fractures. European radiology. $2005 ; 15(1): 165-169$. 
8. Eggenberger E, Hildebrand G, Vang S, Ly A, Ward C. Use of CT Vs. MRI for Diagnosis of Hip or Pelvic Fractures in Elderly Patients After Low Energy Trauma. Iowa Orthop. J. 2019 ; 39(1) : 179-183.

9. Kirchgesner T, Tamigneaux C, Acid S, Perlepe V, Lecouvet F, Malghem J, Berg BV. Fasciae of the muscloskeletal system: MRI findings in trauma, infection and neoplastic diseases. Insights Imaging. 2019 Dec ; 10 : 47. Published online 2019 Apr 18. doi : 10.1186/s13244-0190735-5

10. Cosker T, Ghandour A, Gupta S, Tayton K. Pelvis ramus fractures in the elderly : 50 paitents studied with MRI. Acta Orthop. 2005 Aug ; 76(4) : 513-6.

11. T. Omura, M. Takahashi, Y. Koide, T. Ohishi, A. Yamanashi, K. Kushida, T. Inoue. Evaluation of isolated fractures of the greater trochanter with magnetic resonance imaging. Archives of Orthopaedic and Trauma Surgery, Feb 2000 ; 120 (3-4) : 195-197.

12. Frieda Feldman, Ronald B. Staron. MRI of Seemingly Isolated Greater Trochanteric Fractures. American Journal of Roentgenology. 2004 ; 183 : 323-329.

13. P. Cserhátie, G. Kazár, .J. Manninger, K. Fekete, S. Frenyó. Non-operative or operative treatment for undisplaced femoral neck fractures : a comparative study of 122 non-operative and 125 operatively treated cases. Injury, 1996 Oct. ; 27(8), : 583-588.

14. P. F. Rizzo, E. S. Gould, J. P. Lyden, S. E. Asnis. Diagnosis of occult fractures about the hip. Magnetic resonance imaging compared with bone-scanning, Journal of Bone and Joint Surgery A, 1993 ; 75(3) : 395-401.

15. Je S, Kim H, Ryu S, Cho S, Oh S, Kang T, Choi S. The Consequence of Delayed Diagnosis of an Occult Hip Fracture. Journal of Trauma and Injury 2015 ; 28 : 91-97.

16. Kee-Haeng Lee, Hyoung-Min Kim, Youn-Soo Kim, Changhoon Jeong, Chan-Woong Moon, Sang-Uk Lee, Il-Jung Park. Isolated fractures of the greater trochanter with occult intertrochanteric extension. Archives of Ortho-paedic and Trauma Surgery. October $2010 ; 130$ (10) : 1275-1280.

17. LaLonde B, Fenton P, Campbell A, Wilson P, Yen D. Immediate weight-bearing in suspected greater trochanter fractures as delineated on MRI. The Iowa Orthopaedic Journal. 2010 ; 30 : 201-204.
18. Scheyerer $\mathbf{M J}^{1}$, Osterhoff G, Wehrle S, Wanner GA, Simmen HP, Werner CM. Detection of posterior pelvic injuries in fractures of the pubic rami. Injury. 2012 Aug; 43(8) : 1326-9.

19. O'Connor TJ, Cole PA. Pelvic Insufficiency Fractures. Geriatric Orthopaedic Surgery \& Rehabilitation. 2014 ; 5(4) : 178-190.

20. R. Eastell D.M. Reid J. Compston C. Cooper I. Fogelman R.M. FrancisS.M. Hay D.J. Hosking D.W. Purdie S.H. Ralston. Secondary prevention of osteoporosis : when should a non-vertebral fracture be a trigger for action? QJM : An International Journal of Medicine, 2001 1 Nov. ; 94(11) : 575-597.

21. Rommens PM, Wagner D, Hofmann A. Surgical management of osteoporotic pelvic fractures: a new challenge. European Journal of Trauma and Emergency Surgery. $2012 ; 38(5)$ : 499-509.

22. Culemann U, Scola A, Tosounidis G, Pohlemann T, Gebhard F. Concept for treatment of pelvic ring injuries in elderly patients. A challenge. Unfallchirurg. 2010 ; 113 : 258-271.

23. Freire V, Bureau N, Deslandes M, Moser T. Iliopsoas Tendon Tear: Clinical and Imaging Findings in 4 Elderly Patients. Canadian Association of Radiologists Journal. 2013 Aug. ; 64(3) : 187-192.

24. Cvitanic O, Henzie G, Skezas N, Lyons J, Minter J. MRI diagnosis of tears of the hip abductor tendons (gluteus medius and gluteus minimus). AJR Am J Roentgenol. 2004 Jan ; 182(1) : 137-43.

25. Lachiewicz PF. Abductor tendon tears of the hip : evaluation and management. J. Am. Acad. Orthop. Surg. 2011 Jul ; $19(7): 285-91$.

26. Davies JF, Davies DM. Surgical Technique for the Repair of Tears to the Gluteus Medius and Minimus Tendons of the Hip. JBJS Essent. Surg. Tech. 2014 Jun ; 4(2) : e11.

27. Rubio M, Rodriguez M, Patnaik S, Wang P. Spontaneous iliopsoas tendon tear - A rare cause of hip pain in the elderly. Geriatr. Orthop. Surg. Rehabil. 2016 Mar; 7(1) : 30-32.

28. Bui KL, Ilaslan H, Recht M, Sundaram M. Iliopsoas injury : an MRI study of patterns and prevalence correlated with clinical findings. Skeletal. Radiol. 2008 Mar; 37(3) : 245-9. 